\title{
On-line Ramsey Numbers for Paths and Stars
}

\author{
J.A. Grytczuk, ${ }^{1}$ H.A. Kierstead ${ }^{2}$, and P. Prałat ${ }^{3}$ \\ ${ }^{1}$ Algorithmics Research Group, Faculty of Mathematics and Computer Science, Jagiellonian University, 30-387 \\ Kraków, Poland, \\ ${ }^{2}$ Department of Mathematics and Statistics, Arizona State University, Tempe, Arizona 85287, U.S.A. \\ ${ }^{3}$ Department of Mathematics and Statistics, Dalhousie University, Halifax NS, Canada B3H 3J5
}

received Feb 8, 2007, revised Jul 21, 2008, accepted Sep 8, 2008.

\begin{abstract}
We study on-line version of size-Ramsey numbers of graphs defined via a game played between Builder and Painter: in one round Builder joins two vertices by an edge and Painter paints it red or blue. The goal of Builder is to force Painter to create a monochromatic copy of a fixed graph $H$ in as few rounds as possible. The minimum number of rounds (assuming both players play perfectly) is the on-line Ramsey number $\widetilde{r}(H)$ of the graph $H$. We determine exact values of $\widetilde{r}(H)$ for a few short paths and obtain a general upper bound $\widetilde{r}\left(P_{n}\right) \leq 4 n-7$. We also study asymmetric version of this parameter when one of the target graphs is a star $S_{n}$ with $n$ edges. We prove that $\widetilde{r}\left(S_{n}, H\right) \leq n \cdot e(H)$ when $H$ is any tree, cycle or clique.
\end{abstract}

Keywords: size Ramsey number, online Ramsey games

\section{Introduction}

In this paper we are concerned with on-line variant of size-Ramsey numbers of graphs. For given graphs $G$ and $H$ we write $G \rightarrow H$ if there is a monochromatic copy of $H$ in any red-blue coloring of the edges of $G$. The size-Ramsey number of a graph $H$, denoted by $\widehat{r}(H)$, is the smallest possible number of edges in a graph $G$ satisfying $G \rightarrow H$. This notion was first studied by Erdôs, Faudree, Rousseau, and Schelp (8), as a variation on the usual Ramsey number $r(H)$ (which is the least $n$ such that $K_{n} \rightarrow H$, where $K_{n}$ is a clique on $n$ vertices). Clearly, $\widehat{r}(H)$ is at most $\left(\begin{array}{c}r(H) \\ 2\end{array}\right)$ for every graph $H$. Somewhat surprisingly this bound is attained if $H=K_{n}(8)$. However, $\widehat{r}\left(P_{n}\right) \leq c n$ for some absolute constant $c$, where $P_{n}$ is a path on $n$ vertices. This was proved by Beck (2) in response to a one-hundred-dollar question of Erdôs. The proof uses random graphs and is nonconstructive (cf. (5)). Explicit construction based on expanders was provided by Alon and Chung (1). Since then many deep results for other natural classes of graphs were obtained (cf. $(3 ; 7 ; 15 ; 22))$.

On-line version of size-Ramsey numbers was introduced independently by Beck (4) and Kurek and Ruciński (14). It is best explained by the following game between Builder and Painter, played on a large set of vertices. In one round Builder joins two nonadjacent vertices by an edge and Painter colors this edge red or blue. The goal of Builder is to create a monochromatic copy of a fixed graph $H$ in as few rounds as possible. Painter will try to resist doing it for as long as possible. The on-line Ramsey number 1365-8050 (c) 2008 Discrete Mathematics and Theoretical Computer Science (DMTCS), Nancy, France 
$\widetilde{r}(H)$ of a graph $H$ is the minimum number of rounds in which Builder achieves his goal, assuming both players play perfectly. Clearly, $\widetilde{r}(H) \leq \widehat{r}(H)$ and it is natural to ask how much these two parameters may differ. We study this question in several typical situations. For instance we show that $\widetilde{r}\left(P_{n}\right) \leq 4 n-7$, while currently best bound in the off-line case is $\widehat{r}\left(P_{n}\right) \leq 594 n$ (cf. (5)). In general the problem may be hard as it is not even known if the ratio $\widetilde{r}\left(K_{n}\right) / \widehat{r}\left(K_{n}\right)$ tends to zero as $n \rightarrow \infty$ (cf. (14)). Other open problems and related versions of the game can be found in $(10 ; 12 ; 13)$.

One can also consider an asymmetric version of the on-line Ramsey number. We write $F \rightarrow(G, H)$ if in any red-blue coloring of the edges of $F$ there is a red copy of $G$ or a blue copy of $H$. Similarly, $\widehat{r}(G, H)$ stands for the minimum number of edges in a graph $F$ such that $F \rightarrow(G, H)$, and $\widetilde{r}(G, H)$ is defined accordingly.

Similar to the classical Ramsey numbers (see a dynamic survey of Radziszowski (21) which includes all known nontrivial values and bounds for Ramsey numbers), it is hard to compute the exact value of $\widetilde{r}(G)$ unless $G$ are trivial. In this relatively new area of small on-line Ramsey numbers, very little is known.

\section{Paths}

As a warm-up we start with exact determination of the on-line Ramsey numbers for a few short paths. Let $e(H)$ denote the number of edges of a graph $H$. Note first that in general we have $\widetilde{r}(H) \geq 2 e(H)-1$, since Painter may color safely the first $e(H)-1$ edges red, and the next $e(H)-1$ edges blue. Since $e\left(P_{n}\right)=n-1$ we have $\widetilde{r}\left(P_{n}\right) \geq 2 n-3$. This lower bound is attained for $n=2,3,4,5$.

Proposition $2.1 \widetilde{r}\left(P_{n}\right)=2 n-3$, for $n=2,3,4,5$.

The cases $n=2,3$ are trivial. The case $n=4$ is also very easy; after presenting three edges of a path $P_{4}$, there are only two possible patterns (up to symmetry): $b b r$ and $b r b$. Then Builder creates a monochromatic path $P_{4}$ in the next two moves, as depicted in Figure 1. (The final edge is drawn in two colors.) To prove that $\widetilde{r}\left(P_{5}\right)=7$ we have to analyze more subcases. In the first four moves Builder constructs a path $P_{5}$ so that essentially one of the three possible color patterns appears, as displayed in Figure 2. Then he obtains a monochromatic $P_{5}$ in next three moves, as shown in Figure 3. (A circled number means that Painter had a choice in that move, which led to a branching into subcases.)

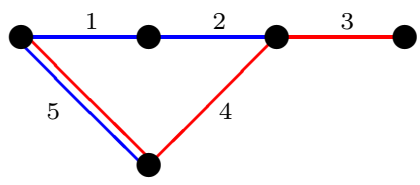

(a) Case 1

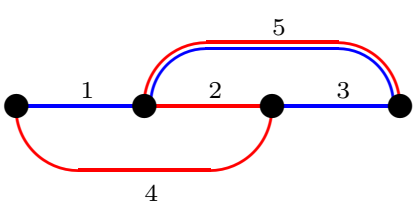

(b) Case 2

Fig. 1: Forcing a path $P_{4}$

The pattern breaks already for $n=6$.

Proposition $2.2 \widetilde{r}\left(P_{6}\right)=10$. 


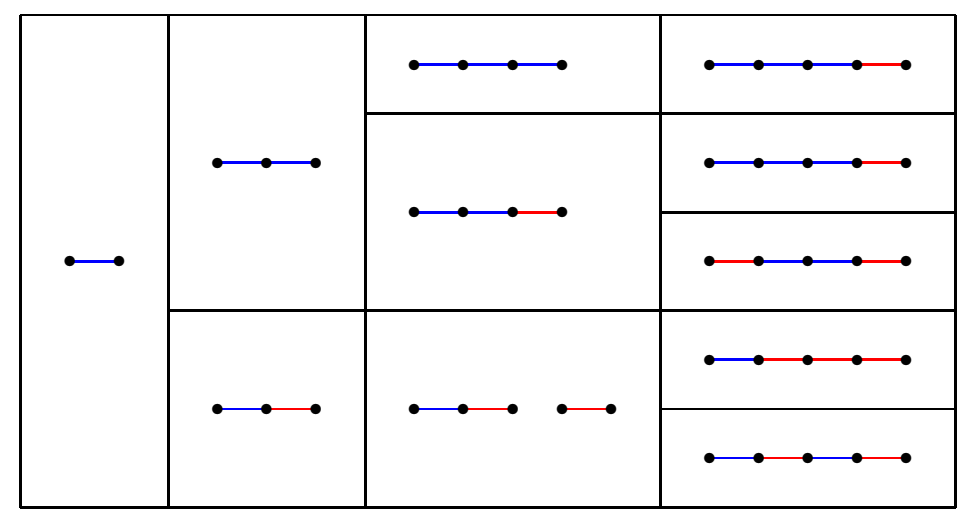

Fig. 2: Forcing a monochromatic $P_{5}$ (first four moves)

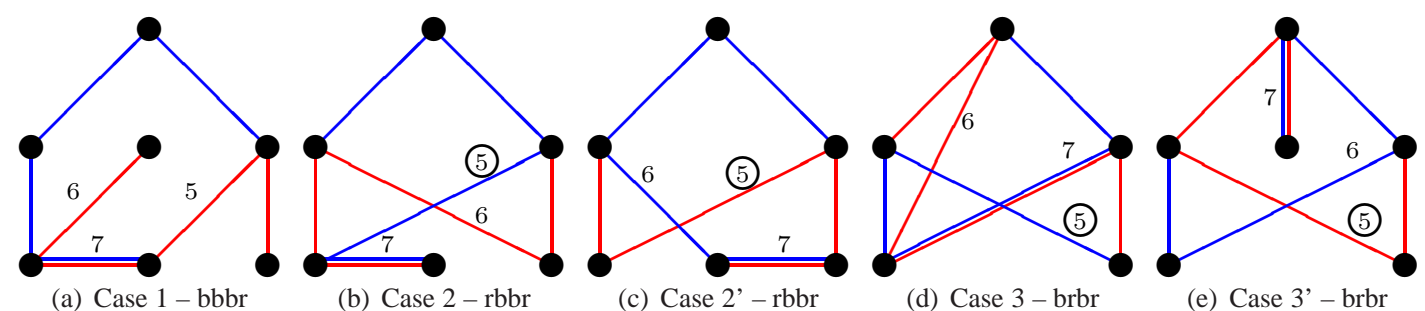

Fig. 3: Forcing a path $P_{5}$ (last three moves) 
Proof: For the lower bound consider a natural strategy for Painter: color an edge blue if it does not create a blue copy of $P_{6}$, otherwise use red. Suppose first that the graph constructed by Builder in the first four moves is not a path $P_{5}$. Then clearly Painter will be able to use the color blue in the very next move and we are done. So, suppose that after first four rounds there is a blue path $P_{5}$. The only way for Builder to finish the game in the total of nine rounds is to create in next four rounds a red $P_{5}$. This is possible only by using both ends of the blue path $P_{5}$, making the last winning move impossible.

The upper bound is achieved by case-by-case analysis shown in Figure 4 (first five moves) and Figure 5 (last five moves).

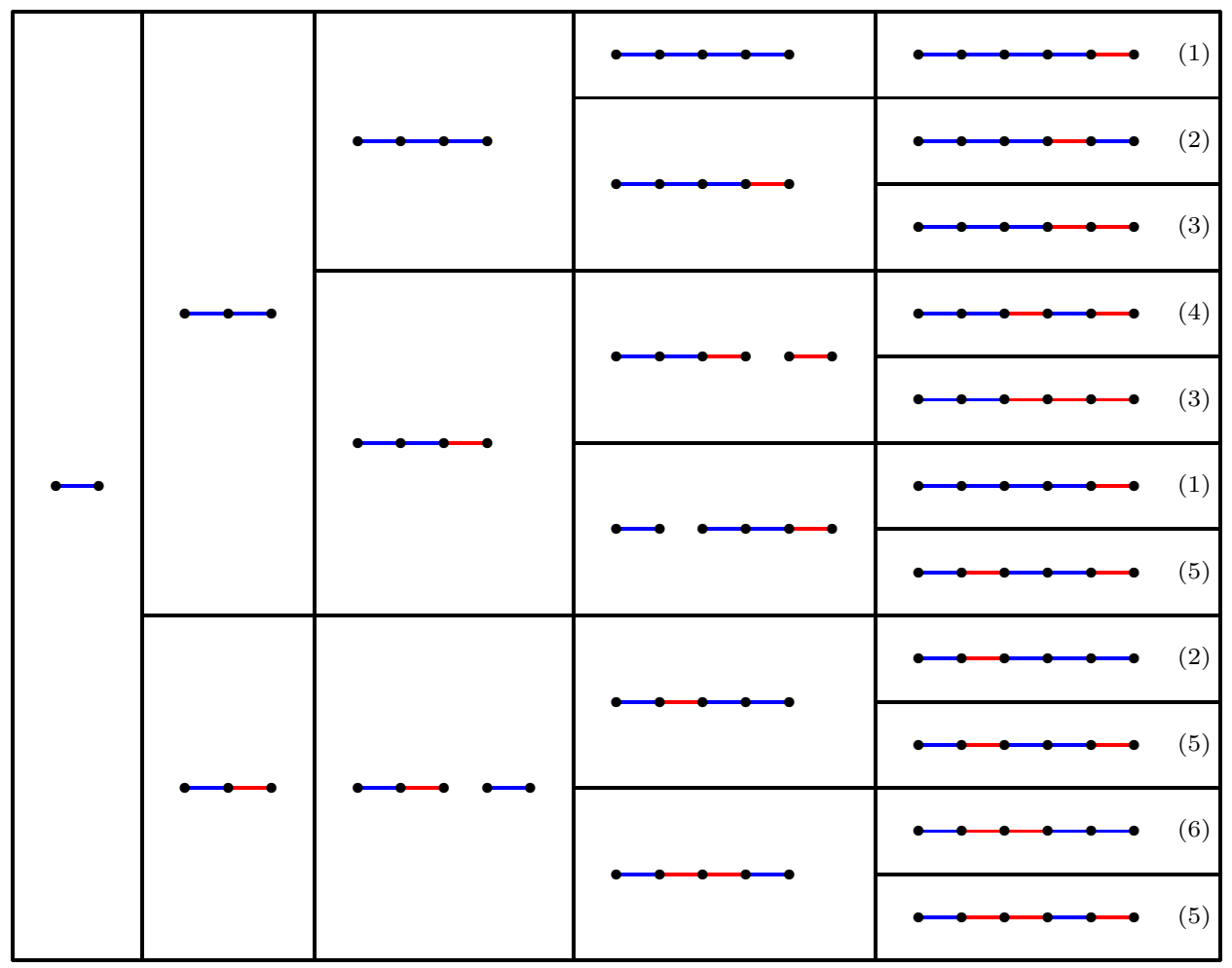

Fig. 4: Forcing a monochromatic $P_{6}$ (first five moves)

Further computer assisted results for small paths can be found in (18; 19). (It has been shown that $\widetilde{r}\left(P_{7}\right)=12, \widetilde{r}\left(P_{8}\right)=15$, and $\widetilde{r}\left(P_{9}\right)=17$. The values for an asymmetric version of the on-line Ramsey number for paths of lengths at most 8 are also determined.) In general we have the following upper bound.

Theorem 2.3 Let $k \geq 1$. In $2 k-1$ rounds Builder can force Painter to create two vertex disjoint monochromatic paths (the red one and the blue one) the sum of whose lengths is equal to $k$.

In particular, $\widetilde{r}\left(P_{n}\right) \leq 4 n-7$ for all $n \geq 2$. 


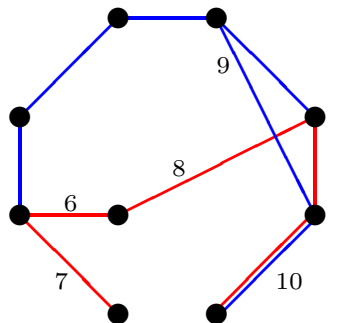

(a) Case $1-$ bbbbr

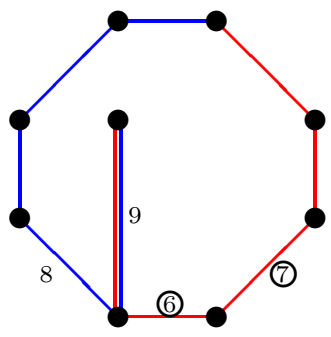

(e) Case $3 b-$ bbbrr

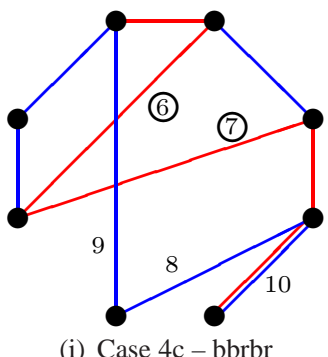

(i) Case $4 \mathrm{c}-$ bbrbr

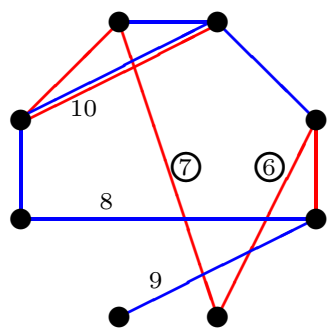

(m) Case $5 d-$ brbbr

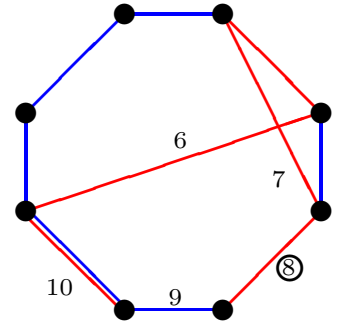

(b) Case $2 \mathrm{a}-\mathrm{bbbrb}$

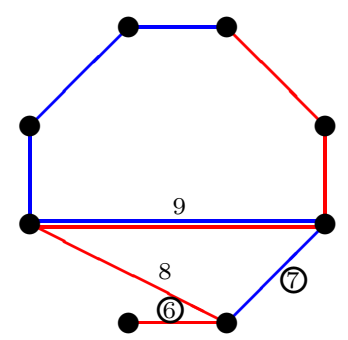

(f) Case 3c-bbbrr

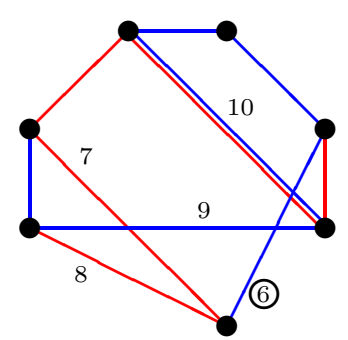

(j) Case 5a-brbbr

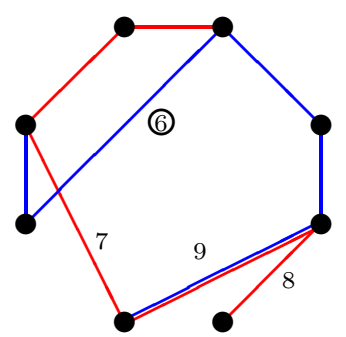

(n) Case 6a-brrbb

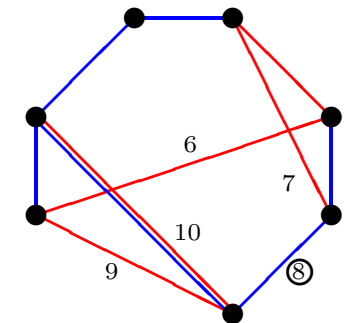

(c) Case $2 b-b b b r b$

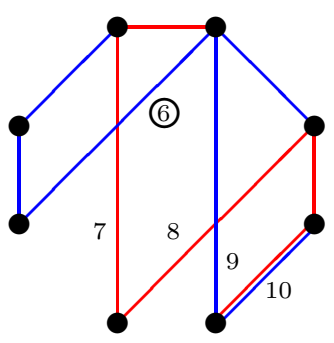

(g) Case $4 a-b b r b r$

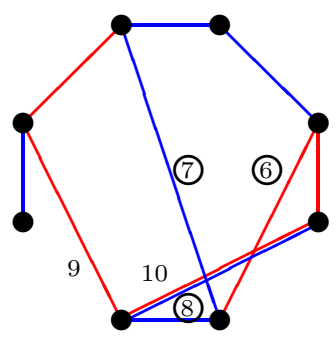

(k) Case $5 \mathrm{~b}-$ brbbr

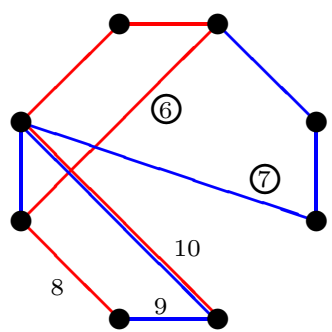

(o) Case $6 b-$ brrbb

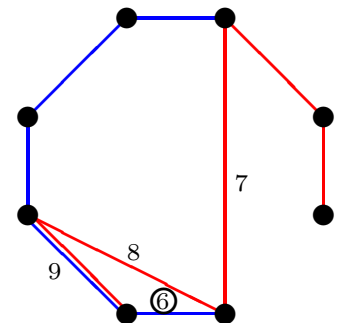

(d) Case $3 \mathrm{a}-$ bbbrr

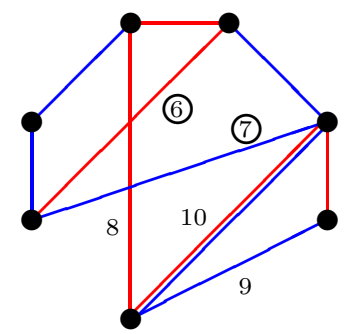

(h) Case $4 b-$ bbrbr

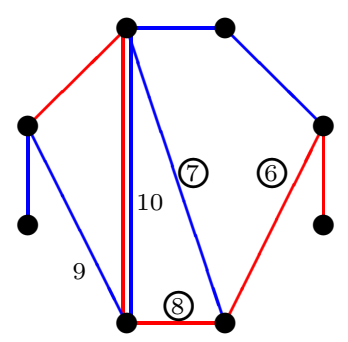

(1) Case $5 c-$ brbbr

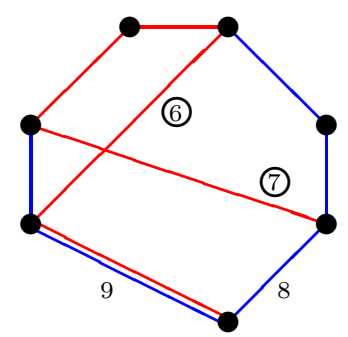

(p) Case $6 c-$ brrbb

Fig. 5: Forcing a path $P_{6}$ (last five moves) 
Proof: The proof goes by induction on $k$. The basis step $k=1$ is trivial. For the inductive step assume that the statement holds for some $k \geq 1$, that is after $2 k-1$ rounds there is a red path $R$ and a blue path $B$ such that $V(R) \cap V(B)=\emptyset$ and $|E(R) \cup E(B)|=k$. Let $v_{R}$ and $v_{B}$ denote any end vertices of $R$ and $B$, respectively (take any isolated vertex as $v_{R}$ or $v_{B}$ if the corresponding path has no edge). If there is no edge $v_{R} v_{B}$, then Builder draws the edge $v_{R} v_{B}$ and Painter does her job (note that it is only to Builder's advantage if it already exists).

Suppose Painter colors this edge red. Then Builder draws an edge from vertex $v_{B}$ to a new vertex $v$. If Painter uses red color, then there are two vertex disjoint monochromatic paths $R^{\prime}=R+v_{B}+v$ and $B^{\prime}=B-v_{B}$, with $k+1$ edges in total. If Painter colors $v_{B} v$ blue, the paths $R^{\prime}=R$ and $B^{\prime}=B+v$ satisfy the assertion. If initially Painter colors the edge $v_{B} v_{R}$ blue, then Builder draws an edge from $v_{R}$ to a new vertex $v$ instead, and the situation is symmetric.

In order to finish the proof notice that after $4 n-7=2(2 n-3)-1$ rounds there must be a monochromatic path with at least $n-1$ edges, that is, a monochromatic $P_{n}$.

\section{Trees}

Let $f(n)$ be the maximum value of $\widetilde{r}(T)$ taken over all trees with $n$ edges. In (9) Erdôs and Graham proved that $r(T) \leq 4 e(T)+1$ for every tree $T$. From this it follows that $f(n)=O\left(n^{2}\right)$. We show that this bound cannot be improved asymptotically, that is, $f(n)=\Theta\left(n^{2}\right)$. First we derive a general lower bound for $\widetilde{r}(H)$ for arbitrary graphs and then present an example that proves the statement. Let $\tau(H)$ denote the smallest number of vertices covering all edges of $H$. The size-Ramsey number of $H$ can be bounded from below in terms of $\tau(H)$ and the maximum degree $\Delta(H)$ of a graph $H$. A similar bound holds in the on-line case.

Proposition $3.1 \widetilde{r}(H) \geq \frac{1}{2} \tau(H)(\Delta(H)-1)+e(H)$ for every graph $H$.

Proof: Painter's strategy is to color an edge red as long as the red subgraph has maximum degree strictly less than $\Delta(H)$; otherwise she colors an edge blue. So, a red copy of $H$ will never appear. Suppose a blue copy of $H$ has appeared in a graph constructed by Builder. Then at least one end vertex of each blue edge must be incident with exactly $\Delta(H)-1$ red edges. Hence the red subgraph must have at least $\tau(H)$ vertices of degree $\Delta(H)-1$ and thus the number of red edges is at least $\frac{1}{2} \tau(H)(\Delta(H)-1)$.

Now, we are ready to show that $f(n)=\Omega\left(n^{2}\right)$. Let $B_{n}$ be a graph with $n$ edges, obtained from a star $S_{\lfloor n / 2\rfloor}=K_{1,\lfloor n / 2\rfloor}$ by subdividing each edge, and with an additional edge attached to the center of the star if $n$ is odd.

Corollary $3.2 f(n) \geq \widetilde{r}\left(B_{n}\right) \geq \frac{n^{2}}{8}+\frac{3 n}{4}$, for every $n \geq 1$.

Proof: We have $\Delta\left(B_{n}\right)=\tau\left(B_{n}\right)=\lceil n / 2\rceil$ as each cover must contain a non-center vertex from each subdivided edge and one extra vertex for an additional edge if $n$ is odd.

If Builder knows that Painter will follow the strategy from Proposition 3.1, then he can force $B_{n}$ in $\left(\begin{array}{c}\lceil n / 2\rceil \\ 2\end{array}\right)+n=\frac{n^{2}}{8}+O(n)$ moves. He starts by building a $\lceil n / 2\rceil$-clique, which Painter colors entirely red. From now on, Painter will color any vertex incident to a clique vertex blue. So Builder can force a blue $B_{n}$ in $n$ moves by using the clique vertices as subdivision vertices of $B_{n}$. Painter can delay her agony a 
little by coloring edges that are incident to the clique and do not create forbidden graph with red, but her real problem was allowing the red clique.

On the other hand, at most $\frac{n^{2}}{4}+O(n)$ moves are enough to force Painter to create monochromatic $B_{n}$.

Proposition $3.3 \widetilde{r}\left(B_{n}\right) \leq \frac{n^{2}}{4}+\frac{9}{2} n-7$ for every even $n=2 k \geq 4$.

Proof: Builder starts by playing $6 k-5$ independent edges. Painter is forced to create a monochromatic matching $M=\left\{x_{i} y_{i}: i \in[3 k-2]\right\}$; suppose the edges of $M$ are blue. Add a new vertex $v$ and edges $v x_{i}$ for $i \in[3 k-2]$. To avoid creating $B_{n}$, Painter must color $2 k-1$ of these edges with red. Say she colors $x_{i} y_{i}$ red for $i \in[2 k-1]$. In the next $k^{2}$ steps, Builder introduces $k$ new vertices $z_{l}(l \in[k])$ and edges $z_{l} x_{m}$ (for some values of $m$ ), forcing Painter to either create a blue star with center at some $z_{l}$ (thus finishing a blue $B_{n}$ ), or a red matching $M^{\prime}=\left\{z_{l} x_{m_{l}}: l \in[k]\right\}$ (thus finishing a red $B_{n}$ ). To do this, he simply plays $k$ edges between $z_{l}$ and $k$ vertices of the form $x_{m}$, where $x_{m}$ is not incident with an edge in $M^{\prime}$.

This takes $(6 k-5)+(3 k-2)+k^{2}$ edges. Builder can save some edges by being more careful at the beginning.

If we restrict to trees of bounded degree then even the size-Ramsey numbers are linearly bounded (cf. (11)).

\section{Stars versus trees, cycles, and cliques}

In this section we focus on asymmetric version of the on-line Ramsey number where one of the graphs is a star $S_{n}$.

It is clear that the Proposition 3.1 can be easily generalized to the asymmetric version we consider. Since the proof is almost identical, we omit it here.

Proposition 4.1 $\widetilde{r}(G, H) \geq \frac{1}{2} \max \{\tau(H)(\Delta(G)-1)+e(H), \tau(G)(\Delta(H)-1)+e(G)\}$ for arbitrary graphs $G$ and $H$.

In particular, $\widetilde{r}\left(S_{n}, H\right) \geq \frac{1}{2} \tau(H)(n-1)+e(H)$.

Before we state a general upper bound we need a few more definitions. Let $\pi$ be a permutation of the vertices of $G$. For a vertex $v \in V(G)$, let $N_{\pi}^{+}(v)$ be the number of neighbors of $v$ that follow it in the permutation. Similarly, we define $N_{\pi}^{-}(v)$. Let $d_{\pi}(G)$ be the maximum "back degree", that is,

$$
d_{\pi}(G)=\max \left\{N_{\pi}^{-}(v): v \in V(G)\right\},
$$

and $l_{\pi}(G)$ be the number of vertices with no "forward neighbors", that is,

$$
l_{\pi}(G)=\sum_{v \in V(G)} \delta\left(N_{\pi}^{+}(v)=0\right)
$$

where $\delta(A)$ is the Kronecker delta function

$$
\delta(A)= \begin{cases}1, & \text { if } A \text { is true } \\ 0, & \text { otherwise. }\end{cases}
$$

Finally, let $n(G)$ denote the number of vertices in a graph $G$. 
Proposition 4.2 $\widetilde{r}\left(S_{n}, H\right) \leq \min _{\pi} d_{\pi}(H)\left(n(H)-l_{\pi}(H)\right)(n-1)+e(H)$ for any graph $H$.

Proof: Fix a permutation $\pi$ of vertices of $H$. We present Builder's strategy to construct $H$ in an order yielded by permutation $\pi$. When we consider a new vertex $v$ of $H$, we choose a new vertex $w$ in the graph we are constructing and add all edges from $w$ to previously assigned vertices that correspond to the neighbors of $v$ in $H$. If Painter uses blue color only, we assign $w$ to $v$ and move to the next vertex in $H$. Otherwise we try again starting from a new vertex $w$. In total we can have at most $\left(n(H)-l_{\pi}(H)\right)(n-1)$ retries since that is exactly an upper bound for the number of red edges when Painter is trying to avoid red $S_{n}$ and each retry can be associated with a unique red edge generated in the previous try. In each retry we use at most $d_{\pi}(H)$ edges.

Now, we are ready to prove bounds for $\widetilde{r}\left(S_{n}, H\right)$ in a few special cases where graph $H$ is any tree, cycle or clique.

Proposition 4.3 Let $T$ be any tree with $e(T) \geq 2$ edges and $l$ vertices of degree 1 (leaves). Then for every $n \in \mathbb{N}$

$$
\widetilde{r}\left(S_{n}, T\right) \leq e(T) n-(l-1)(n-1) .
$$

The proof follows immediately from Proposition 4.2 since there is a permutation $\pi$ with $d_{\pi}(G)=1$ and $l_{\pi}(G)=l$. We present an alternative proof below.

Proof: Let $r \in V(T)$ be any vertex of degree at least 2 , and select this vertex as a root of the tree $T$ making this tree a rooted one. Let $d(v)$ denote the degree of a vertex $v$ in the tree $T$. In the first $d(r)+n-1$ moves Builder draws a star $S_{d(r)+n-1}$ forcing Painter to create a blue star $S_{d(r)}$. This is the first level of $T$. Then for every leaf $v$ of the blue star $S_{d(r)}$, which is not a leaf of $T$, Builder draws a star $S_{(d(v)-1)+(n-1)}$ with center in $v$. This creates the second level of $T$ (see Figure 6). And so on, until a complete blue copy of $T$ (or a red copy of $S_{n}$ ) appears. The total number of edges drawn by Builder is

$$
(n-1)(|V(T)|-l)+e(T)=e(T) n-(l-1)(n-1)
$$

as asserted.

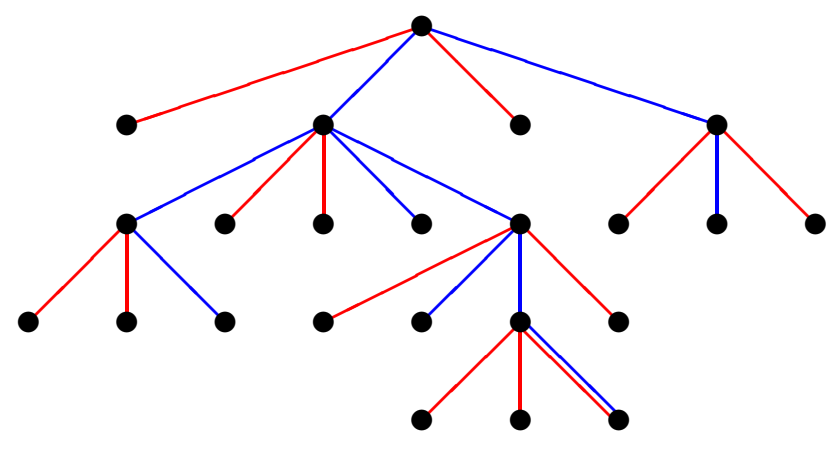

Fig. 6: Forcing a red $K_{1, n}$ or blue $T$

In next proposition we show that the on-line Ramsey number for a star $S_{n}$ versus a fixed cycle grows linearly in $n$. This is different than in the off-line case, where for odd cycles we have a quadratic growth (cf. $(16 ; 17)$ ). 
Proposition 4.4 For every $n, k \in \mathbb{N}, k \geq 3$

$$
\frac{k}{4}(n+3) \leq \widetilde{r}\left(S_{n}, C_{k}\right) \leq n k .
$$

Proof: The lower bound follows immediately from Proposition 4.1 since $\tau\left(C_{k}\right) \geq k / 2$.

For the upper bound, Builder first forces a blue path with $k-2$ edges. By Proposition 4.3 this takes at most $n(k-3)+1$ rounds. Let $v_{1}$ and $v_{k-1}$ be the two end vertices of this path. Builder draws a star $S_{2 n-1}$ with center in $v_{k-1}$ and new vertices $u_{1}, \ldots, u_{2 n-1}$ as its leaves. Clearly at least $n$ edges of this star must be blue. Then he joins these vertices to the vertex $v_{1}$. If there is no red star yet, then there must be a vertex $u_{i}$ such that both edges $u_{i} v_{1}$ and $u_{i} v_{k-1}$ are blue. Hence a blue cycle is created (see Figure 7). Total number of edges is equal to $((k-3) n+1)+(2 n-1)+n=k n$.

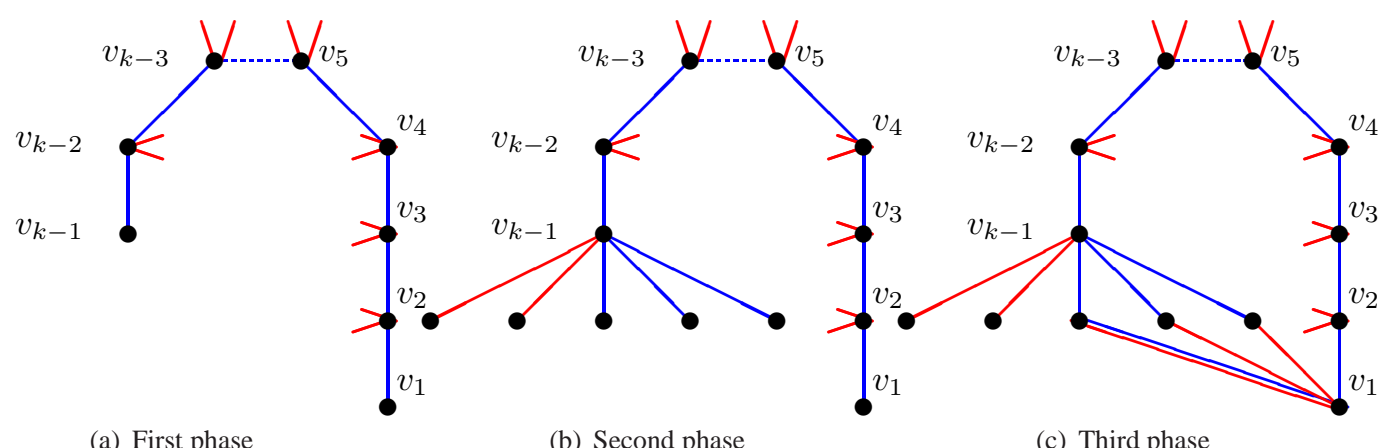

Fig. 7: Forcing a red $K_{1, n}$ or blue $C_{k}$

It is clear that better bounds can be achieved with more effort. For example let us mention the following small improvement. If Painter creates red $S_{n-1}$ in the first phase, then Builder can start growing a path from the other direction so that vertex $v_{1}$ is attached to the red star. Now in the second phase we can generate one blue edge only (instead of $n$ as before). This saves at least $\min \{n-1, k-3\}$ edges. Since there is no hope for a general exact formula, we are happy with the bound which is optimal up to a multiplicative constant.

A similar bounds hold for cliques $K_{k}$. The bound is optimal up to a factor of $c k$ for some constant $c>0$.

Proposition 4.5 For every $n, k \in \mathbb{N}, k \geq 2$

$$
\frac{1}{2}(k-1)(n-1)+\left(\begin{array}{l}
k \\
2
\end{array}\right) \leq \widetilde{r}\left(S_{n}, K_{k}\right) \leq\left(\begin{array}{l}
k \\
2
\end{array}\right) n .
$$

Proof: Again, the lower bound is a simple consequence of Proposition 4.1 since $\tau\left(K_{k}\right)=k-1$.

For the upper bound, let $V_{0}$ be a set of $\left|V_{0}\right|=(k-1) n+1$ vertices. First Builder proposes $(k-1) n$ edges of a star $S_{(k-1) n}$ on the set $V_{0}$. Since Painter is trying to avoid a red star with $n$ edges, at least $(k-2) n+1$ edges must be colored blue. Let $V_{1} \subset V_{0},\left|V_{1}\right|=(k-2) n+1$ be a set of vertices of degree 
1 connected with a blue edge. In the second phase Builder is drawing a star $S_{(k-2) n}$ on $V_{1}$ forcing Painter to use blue color at least $(k-3) n+1$ times. Thus, there is a set $V_{2} \subset V_{1},\left|V_{2}\right|=(k-3) n+1$ of vertices of degree 2 incident with a blue edges only. Repeating this strategy in each phase, Builder eventually forces Painter to draw either a red star $S_{n}$ or a blue clique $K_{k}$. An example of the game in case of $K_{1,2}$ and $K_{4}$ is shown on Figure 8. Note that the game consists of $k-1$ phases and during the $i$ th round, the Builder is drawing a star with $(k-i) n$ edges. Thus

$$
\widetilde{r}\left(S_{n}, K_{k}\right) \leq \sum_{i=1}^{k-1}(k-i) n=\frac{k(k-1)}{2} n
$$

and the assertion follows.

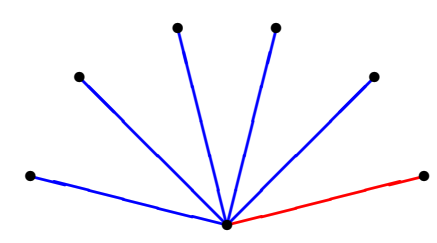

(a) First phase

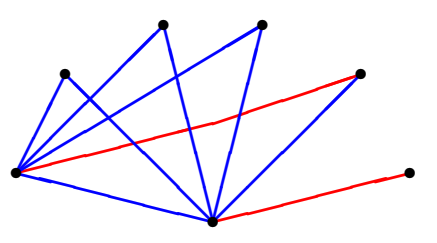

(b) Second phase

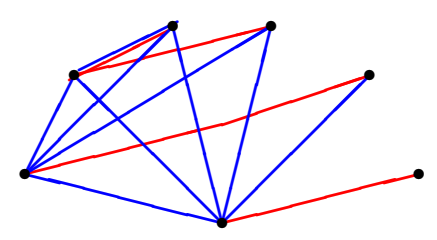

(c) Third phase

Fig. 8: Forcing a red $K_{1, n}$ or blue $K_{k}$

The above results suggest that perhaps $\widetilde{r}\left(S_{n}, H\right) \leq n \cdot e(H)$ for every graph $H$.

\section{Discussion}

We conclude the paper with some questions for future consideration. Let $\mathcal{F}$ be a fixed family of graphs and let $\widetilde{r}(n)=\max \{\widetilde{r}(H): H \in \mathcal{F},|V(H)|=n\}$. Also let $\widehat{r}(n)$ and $r(n)$ be defined analogously for size-Ramsey and Ramsey numbers of graphs from $\mathcal{F}$, respectively. Clearly for any class $\mathcal{F}$ we have

$$
\widetilde{r}(n) \leq \widehat{r}(n) \leq\left(\begin{array}{c}
r(n) \\
2
\end{array}\right) .
$$

A general problem is to determine the asymptotic growth of the function $\widetilde{r}(n)$ for some basic graph classes. In particular, to decide whether

$$
\lim _{n \rightarrow \infty} \frac{\widehat{r}(n)}{\widetilde{r}(n)}=\infty .
$$

It is natural to expect that (1) should hold for most reasonable families of graphs. Clearly (1) cannot hold if $\widehat{r}(n)$ is linear, but, as we demonstrated, it also does not hold for trees, where $\widehat{r}(n)$ grows quadratically. We do not know what happens for graphs of bounded degree. A well known theorem of Chvátal, Rödl, Szemerédi, and Trotter (6) asserts that $r(n)$ grows linearly for every fixed $\Delta$ (hence $\widehat{r}(n)$ is at most quadratic). On the other hand, as proved by Rödl and Szemerédi (22), there are cubic graphs with quadratic size-Ramsey number. 
It is obvious that the real challenge is in computing the on-line Ramsey numbers for cliques. Therefore, the following intriguing question was posed by Kurek and Ruciński (14): does (1) hold for the family of all cliques? A natural strategy for Builder is to mimic a recursive construction giving the upper bound for classical Ramsey number $r\left(K_{n}\right)$. This gives for instance $\widetilde{r}\left(K_{3}\right) \leq 8$ (in fact, $\widetilde{r}\left(K_{3}\right)=8$; the proof of the lower bound is elementary but nontrivial). Moreover, it has been shown that $\widetilde{r}\left(K_{k}\right) \leq 2 k\left(\begin{array}{c}2 k-2 \\ k-1\end{array}\right) \sim$ $\frac{1}{2 \sqrt{\pi}} \sqrt{k} 4^{k}$. The third author of this note, with computer support, showed that an asymmetric version of the on-line Ramsey number $\widetilde{r}\left(K_{3}, K_{4}\right)$ is equal to 17 , provided a general upper bound for $\widetilde{r}\left(K_{k}, K_{l}\right)$, which gives a slightly better asymptotic upper bound of $\frac{3}{8 \sqrt{\pi}} \frac{4^{k}}{\sqrt{k}}$ for a symmetric version of the on-line Ramsey numbers (see (20) for more details).

\section{References}

[1] N. Alon and F.R.K. Chung, Explicit construction of linear sized tolerant networks, Proceedings of the First Japan Conference on Graph Theory and Applications (Hakone, 1986). Discrete Math. 72 (1988), no. $1-3,15-19$.

[2] J. Beck, On size Ramsey number of paths, trees and cycles I, J. Graph Theory 7 (1983) 115-130.

[3] J. Beck, On size Ramsey number of paths, trees and cycles II. Mathematics of Ramsey Theory, Springer, Berlin, Alorithms and Combin. 5 (1990), 34-45.

[4] J. Beck, Achievement games and the probabilistic method, in: Combinatorics, Paul Erdős is Eighty, Bolyai Soc. Math. Stud., vol. 1, (1993), 51-78.

[5] B. Bollobás, Random graphs, Academic Press, 1986.

[6] V. Chvátal, V. Rödl, E. Szemerédi, and W. T. Trotter, The Ramsey number of a graph with a bounded maximum degree, J. Combin. Theory Ser. B 34 (1983) 239-243.

[7] J. Donadelli, P.E. Haxell, and Y. Kohayakawa, A note on the size-Ramsey number of long subdivisions of graphs, RAIRO-Inf. Theor. Appl. 39 (2005), 191-206.

[8] P. Erdős, R.J. Faudree, C.C. Rousseau, and R.H. Schelp, The size Ramsey number, Period. Math. Hungar. 9 (1978), 145-161.

[9] P. Erdős and R.L. Graham, On partitions theorems for finite graphs, Infinite and Finite Sets, vol. 1, North-Holland Amsterdam, Colloq. Math. Soc. János Bolyai 10 (1975), 515-527.

[10] E. Friedgut, Y. Kohayakawa, V. Rödl, A. Ruciński, and P. Tetali, Ramsey games against one-armed bandit, Combin. Probab. Comput. 12 (2003), 515-545.

[11] J. Friedman and N. Pippenger, Expanding graphs contain all small trees, Combinatorica 7 (1987), $71-76$.

[12] J.A. Grytczuk, M. Hałuszczak, and H.A. Kierstead, On-line Ramsey Theory, Electronic Journal of Combinatorics 11 (2004), no. 1, Research Paper 60, 10 pp. 
[13] H.A. Kierstead and G. Konjevod, Coloring number and on-line Ramsey theory for graphs and hypergraphs, Combinatorica, accepted.

[14] A. Kurek and A. Ruciński, Two variants of the size Ramsey number, Discuss. Math. Graph Theory 25 (2005), no. 1-2, 141-149.

[15] O. Pikhurko, Asymptotic size Ramsey results for bipartite graphs, SIAM J. Discrete Math. 16 (2002), no. $1,99-113$.

[16] O. Pikhurko, Size Ramsey numbers of stars versus 3-chromatic graphs, Combinatorica 21 (2001), no. $3,403-412$.

[17] O. Pikhurko, Size Ramsey numbers of stars versus 4-chromatic graphs, J. Graph Th. 42 (2003), 220-233.

[18] P. Prałat, A note on small on-line Ramsey numbers for paths and their generalization, Australasian Journal of Combinatorics, 40 (2008), 27-36.

[19] P. Prałat, A note on off-diagonal small on-line Ramsey numbers for paths, Ars Combinatoria, 10pp, accepted.

[20] P. Prałat, $\overline{\mathcal{R}}(3,4)=17$, Electronic Journal of Combinatorics 15 (2008), no. 1, Research Paper 67, $13 \mathrm{pp}$.

[21] S. Radziszowski, Small Ramsey Numbers, Electron. J. Combin. Dynamic Survey DS1, revision \#11 (2006), 60pp.

[22] V. Rödl and E. Szemerédi, On size Ramsey numbers of graphs with bounded maximum degree, Combinatorica 20 (2000), 257-262. 